\title{
Absolute Quantitation of Myocardial Blood Flow in Human Subjects With or Without Myocardial Ischemia Using Dynamic Flurpiridaz F 18 PET
}

\author{
René R.S. Packard ${ }^{1}$, Sung-Cheng Huang ${ }^{2}$, Magnus Dahlbom², Johannes Czernin² ${ }^{2}$ and Jamshid Maddahi ${ }^{1,2}$ \\ ${ }^{1}$ Department of Medicine (Cardiology), University of California, Los Angeles, California; and ${ }^{2}$ Department of Molecular and \\ Medical Pharmacology (Nuclear Medicine), Ronald Reagan UCLA Medical Center, University of California, Los Angeles, California
}

\begin{abstract}
Absolute quantitation of myocardial blood flow (MBF) by PET is an established method of analyzing coronary artery disease (CAD) but subject to the various shortcomings of available radiotracers. Flurpiridaz $F 18$ is a novel PET radiotracer that exhibits properties of an ideal tracer. Methods: A new absolute perfusion quantitation method with flurpiridaz was developed, taking advantage of the early kinetics and high first-pass extraction by the myocardium of this radiotracer, and the first-in-human measurements of MBF performed in 7 healthy subjects and 8 patients with documented CAD. PET images with time-activity curves were acquired at rest and during adenosine stress. Results: In healthy subjects, regional MBF between coronary artery territories did not differ significantly, leading to a mean global MBF of $0.73 \mathrm{~mL} / \mathrm{min} / \mathrm{g}$ at rest and 2.53 $\mathrm{mL} / \mathrm{min} / \mathrm{g}$ during stress, with a mean global myocardial flow reserve (MFR) of 3.70 . CAD vascular territories with $<50 \%$ stenosis demonstrated a mean MBF of 0.73 at rest and 2.02 during stress, leading to a mean MFR of 2.97. CAD vascular territories with $\geq 50 \%$ stenosis exhibited a mean MBF of 0.86 at rest and 1.43 during stress, leading to a mean MFR of 1.86. Differences in stress MBF and MFR between normal and CAD territories, as well as between $<50 \%$ and $\geq 50 \%$ stenosis vascular territories, were significant $(P<0.01)$. Conclusion: Absolute quantitation of MBF in humans with the novel PET radiotracer flurpiridaz is feasible over a wide range of cardiac flow in the presence or absence of stressinducible myocardial ischemia. The significant decrease in stress MBF and ensuing MFR in CAD territories allows a clear distinction between vascular territories exhibiting stress-inducible myocardial ischemia and those with normal perfusion.
\end{abstract}

Key Words: flurpiridaz; PET; MBF; MFR; human

J Nucl Med 2014; 55:1438-1444

DOI: 10.2967/jnumed.114.141093

A

bsolute quantitation of myocardial blood flow (MBF) by PET has a significant role in the clinical evaluation of

Received Apr. 3, 2014; revision accepted Jun. 25, 2014.

For correspondence or reprints contact: Jamshid Maddahi, Department of Medicine (Cardiology) and Department of Molecular and Medical Pharmacology (Nuclear Medicine), Ronald Reagan UCLA Medical Center, University of California, 757 Westwood Blvd., 100 Medical Plaza, Ste. 410, Los Angeles, CA 90095.

E-mail: jmaddahi@mednet.ucla.edu

Published online Jul. 28, 2014.

COPYRIGHT (C 2014 by the Society of Nuclear Medicine and Molecular Imaging, Inc. epicardial and microvascular coronary artery disease (CAD) (1). Although the clinical value of the absolute quantitation of MBF by PET is well recognized, this technique is not often used because of the limitations of currently available radiotracers $(2)$.

PET radiotracers such as ${ }^{82} \mathrm{Rb}$-chloride, ${ }^{13} \mathrm{~N}$-ammonia, and ${ }^{15} \mathrm{O}$ water have been used to quantitate absolute MBF. Because of their short half-lives, ${ }^{15} \mathrm{O}$ is limited to facilities with an on-site cyclotron, and ${ }^{13} \mathrm{~N}$ requires either an on-site or a nearby cyclotron. ${ }^{82} \mathrm{Rb}$ is generator-produced and is approved for the clinical assessment of myocardial perfusion. However, its short half-life affects image quality with noisy time-activity curves, reducing the tracer's ability to quantify myocardial perfusion. A desirable myocardial perfusion agent should have a high first-pass extraction fraction and track regional MBF over a wide range, permitting accurate determination of absolute MBF. The agent should exhibit excellent target-to-nontarget uptake ratios, with high uptake in the myocardium and low uptake or rapid clearance from adjacent organs. Furthermore, it should be available as a unit dose from regional cyclotrons, obviating the need for on-site cyclotrons or costly ${ }^{82} \mathrm{Rb}$ generators (2).

Flurpiridaz is a novel PET myocardial perfusion imaging (MPI) agent labeled with ${ }^{18} \mathrm{~F}$. It is a structural analog of the insecticide pyridaben, a known inhibitor of the nicotinamide adenine dinuclelotide hydrate:ubiquinone oxidoreductase (also known as mitochondrial complex-1) of the electron transport chain (3). Flurpiridaz inhibits mitochondrial complex- 1 by competing for binding with ubiquinone without affecting the viability of cardiomyocytes. This radiotracer exhibits a rapid uptake and slow washout from cardiomyocytes (3). Experimental PET imaging demonstrates a high and sustained cardiac uptake that is proportional to blood flow (4). In rats, the first-pass extraction fraction of flurpiridaz by the myocardium is $94 \%$ (5). The flow-independent extraction fraction of flurpiridaz implies a linear relationship between uptake and MBF, an important attribute for stress MBF measurements (5). In a pig model, flurpiridaz exhibits higher activity ratios of the myocardium versus the blood, liver, and lungs than ${ }^{13} \mathrm{~N}$-ammonia (6). Moreover, flurpiridaz has an excellent correlation with radioactive microspheres in assessing absolute quantitation of regional MBF over flow ranges from 0.1 to $3.0 \mathrm{~mL} / \mathrm{min} / \mathrm{g}(6,7)$. This radiotracer also permits evaluation of myocardial infarction size in rats (8). Importantly, the isotope ${ }^{18} \mathrm{~F}$ has a 110 -min half-life, making delivery of unit doses from regional cyclotrons feasible.

Use of this compound in human studies $(2,9,10)$ demonstrated excellent-quality myocardial images in addition to exhibiting many desirable properties of an ideal myocardial perfusion tracer 
including high myocardial uptake, slow myocardial clearance, and high myocardial-to-background contrast (3-5). In the present study, we sought to perform the absolute quantitation of MBF and derive the myocardial flow reserve (MFR) using this compound in a group of healthy subjects and CAD patients.

Methods to use flurpiridaz for the quantitation of myocardial perfusion have been proposed and validated in a pig model (6) in which a 2-tissue-compartmental model was used to fit myocardial kinetics of 10 or $20 \mathrm{~min}$ and to estimate myocardial perfusion. The modeling approach was similar to the one for ${ }^{13} \mathrm{~N}$ ammonia cardiac PET studies, except labeled metabolites in the blood were ignored and the tracer binding deemed irreversible. This approach is susceptible to potential variations in labeled metabolites in the blood and subject movement, which is a major concern with the high spatial imaging resolution of current PET/CT scanners. Others proposed using a static scan to simplify the quantitation procedure (7). They found that in the pig model the MFR obtained with the static-scan approach matched the value obtained with microspheres, provided the scan time is from 5 to $10 \mathrm{~min}$ and the value is normalized by the blood-pool activity in the 0- to 3-min image. The approach, however, still required the subject to be scanned starting with tracer injection time, and it did not provide accurate values for MBF.

In this study, we use a quantitation method that can provide quantitative MBF using Flurpiridaz F 18. The method uses the early kinetics of myocardial uptake of the tracer. It takes advantage of the high first-pass extraction fraction of the tracer (5) - that is, no compartmental modeling is required and is less dependent on the assumptions necessitated by other proposed approaches discussed above. Because the method depends only on the dynamic images of a short early time $(<2 \mathrm{~min})$, it is not as sensitive to subject movements. The method was applied both to healthy subjects with low likelihood of myocardial ischemia and to $\mathrm{CAD}$ patients with stress-inducible myocardial ischemia.

\section{MATERIALS AND METHODS}

Human subject/patient selection, preparation of Flurpiridaz F 18, and statistical analysis can be found in the supplemental data (available online only at http://jnm.snmjournals.org).

\section{PET Imaging Procedures}

Two flurpiridaz PET imaging sessions were performed in each subject/patient. The first was performed at rest and, either on the same day or on the following day, the PET imaging was repeated under stress with adenosine infused at a rate of $140 \mathrm{mcg} / \mathrm{kg}$ of body weight/min for $6 \mathrm{~min}$, starting at $3 \mathrm{~min}$ before the injection of the tracer. Eleven of the PET studies (6 healthy subjects and 5 CAD patients) were done using a same-day protocol, and 4 (1 healthy subject and 3 CAD patients) were done using a 2-d protocol. For the same-day studies, the rest study was performed first, and the stress study was performed $52.9 \pm 11.2 \mathrm{~min}$ later, as previously reported (10). At $60 \mathrm{~min}$ after the rest injection, the tracer distribution in the heart did not change much over a 5-min period, and the time-activity curve values measured before the stress injection were averaged and subtracted from the stress time-activity curve before the modeling analysis was performed. There was no significant increase in noise in the time-activity curves when using the subtraction procedure in the same-day protocol as compared with the 2-d protocol. For each imaging session, the subject was positioned supine in a PET/CT scanner (Biograph 64; Siemens Inc.). After CT imaging of the chest, flurpiridaz (185 MBq [5 mCi]) was administered intravenously as a slow bolus over $10 \mathrm{~s}$, followed by a 5- to $10-\mathrm{mL}$ saline flush. PET imaging was started concomitantly to flurpiridaz injection, and list-mode data were collected for $10 \mathrm{~min}$. Thereafter, the list-mode data were framed into a scan sequence of $12 \times 10,4 \times 30,1 \times 60$, and $1 \times 300 \mathrm{~s}$. Images were reconstructed using a 2-dimensional ordered-subset expectation maximization iterative algorithm (8 subsets and 21 iterations). No postreconstruction filtering was applied, resulting in a spatial resolution of $6.5 \mathrm{~mm}$ in full width at half maximum.

\section{Image Analysis}

The dynamic transaxial images were first reoriented into shortaxis slices. These were then used to produce dynamic polar maps, where the sampling points were defined on a 6-min summed frame at the end of the acquisition. Regions of interest (ROIs) corresponding to the 3 coronary territories were defined on the dynamic polar maps. A polar map was used to normalize the size and shape of hearts from different individuals and allow ROIs to be obtained more consistently and objectively. Tissue time-activity curves were generated from the dynamic polar maps using these ROIs. A bloodpool time-activity curve was generated by placing a small ROI in the center of the left ventricle of the last frame of the dynamic shortaxis images. This procedure was applied to both the rest and the stress data (11).

\section{Absolute Quantitation of MBF}

Quantitative myocardial perfusion in each perfusion territory was estimated using the tracer uptake kinetics within the first $90 \mathrm{~s}$ after tracer injection. Within this period, the tracer was assumed to be taken up by the myocardium without significant clearance and with few, if any, labeled metabolites contained in the blood during this early period. Therefore, the radioactivity concentration in myocardial segment $i$ can be described by Equation 1 below.

$$
C_{i}(t)=F_{i} \int_{0}^{t} C_{p}(\tau) d \tau+V_{b i} C_{p}(t), \text { for } i=1,2,3,
$$

where the subscripts $i=1,2$, and 3 indicate, respectively, the myocardial activities in the 3 perfusion territories, which are the left anterior descending artery (LAD), left circumflex artery (LCx), and right coronary artery $(\mathrm{RCA}) ; C_{p}(t)$ is the radioactivity concentration in blood; and $V_{b i}$ is the vascular volume in that myocardial segment. Because of the partial-volume effect and the spillover of activities between myocardium and blood pool, the measured radioactivity concentration in the blood pool $\left(C_{p}(t)\right)$ and in the myocardial regions of the 3 perfusion territories $\left(C_{i}(t)\right.$, for $\left.i=1,2,3\right)$ from the dynamic PET images can be described by the following equations.

$$
\begin{aligned}
C_{p}(t)_{\text {measured }}= & R C_{b p} \times C_{p}(t)+\sum_{i=1}^{3} S P F_{m b i} \times C_{i}(t) \\
C_{i}(t)_{\text {measured }}= & R C_{i} \times C_{i}(t)+S P F_{b m i} \times C_{p}(t), \text { for } i=1,2,3 \\
= & R C_{i} \times F_{i} \int_{0}^{t} C_{p}(\tau) d \tau+\left(V_{b}+S P F_{b m i}\right) \times C_{p}(t), \\
& \text { for } i=1,2,3,
\end{aligned}
$$

where $R C$ is the recovery coefficient due to the partial-volume effect (of the myocardium or the blood pool), and $S P F$ is the spillover fraction (the subscript bmi denotes the spillover from blood pool to myocardium region $i$, and $m b i$ denotes the spillover fraction from myocardium segment $i$ to the blood-pool ROI).

Equation 2 was used to fit the measured myocardial time-activity curves. With the approximation that $R C_{b p}=0.95$ and $S P F_{m b i}=0.05 / 3$ ( $i=1,2$, and 3 ), the blood-pool time-activity curve $\left(C_{p}(t)\right)$ was calculated 
from the measured blood-pool time-activity curve and the myocardial time-activity curves directly as

$$
\begin{aligned}
C_{p}(t) & =\frac{1}{R C_{b p}} \times\left[C_{p}(t)_{\text {measured }}-\sum_{i=1}^{3} S P F_{m b i} \times C_{i}(t)\right] \\
& =\frac{1}{0.95} \times\left[C_{p}(t)_{\text {measured }}-\sum_{i=1}^{3}(0.05 / 3) \times C_{i}(t)\right] .
\end{aligned}
$$

Furthermore, the spillover fraction from the blood pool to the myocardium plus the vascular volume was approximated as 1.0 minus the $R C$ of the corresponding myocardial segment. That is,

$$
\left(S P F_{b m i}+V_{b i}\right)=1.0-R C_{i} \text { for } i=1,2, \text { and } 3 .
$$

An iterative procedure in which the measured time-activity curve of each perfusion territory was regressed one at a time, with the estimated $C_{p}(t)$ fixed from a previous iteration. After each round of regression of the measured time-activity curves of the 3 perfusion territories, $C_{p}(t)$ was updated (with Eq. 3). The procedure was then repeated. Usually, the estimated values of $F_{i}$ and $R C_{i}$ converged within approximately 3 iterations.

Global myocardial perfusion $\left(C_{g}(t)_{\text {measured }}\right)$ was estimated from the weighted average of the time-activity curves of the 3 territories (weighted by their approximate areas on the polar map). That is,

$C_{g}(t)_{\text {measured }}=\left(7 C_{1}(t)_{\text {measured }}+5 C_{2}(t)_{\text {measured }}+5 C_{3}(t)_{\text {measured }}\right) / 17$.

The equation used for estimating the global myocardial perfusion is the same as those for each myocardial segment (i.e., Eq. 2), except that the $C_{p}(t)$ estimated at the end of the regression for individual myocardial segment was used for estimating the global myocardial perfusion.

After $F_{i}$ was determined, MBF was obtained by assuming a firstpass extraction fraction of 0.94 (5). That is,

$$
M B F_{\mathrm{i}}=F_{i} / 0.94 \text {. }
$$

The units of $M B F_{i}$ are in ( $\mathrm{mL}$ of blood) $/ \mathrm{min} /(\mathrm{g}$ of myocardium), assuming that the specific density of myocardium is approximately $1.0 \mathrm{~g} / \mathrm{mL}$. Because the kinetic data were corrected for partial volume, spillover, and vascular activity, the MBF value obtained corresponded to the blood perfusion value in the myocardium with blood in the vasculature removed.

\section{Calculation of MFR}

MFR for each myocardial segment was calculated as the ratio of the segment's MBF value with adenosine infusion to the MBF value at rest.

$$
\mathrm{MFR}=(\mathrm{MBF} \text { during adenosine infusion }) /(\mathrm{MBF} \text { at rest })
$$

\section{RESULTS}

A representative polar map depicting relative perfusion in a CAD patient with stress-inducible ischemia is shown, demonstrating a reversible defect mostly in the LAD distribution (Fig. 1).

The tracer kinetics in a normal myocardial region along with the blood-pool time-activity curve is illustrated in Figure 2. The model fitted curves at the end of the MBF estimation are also shown.

The MBF in each vascular territory of healthy subjects with low likelihood of myocardial ischemia under rest and stress conditions

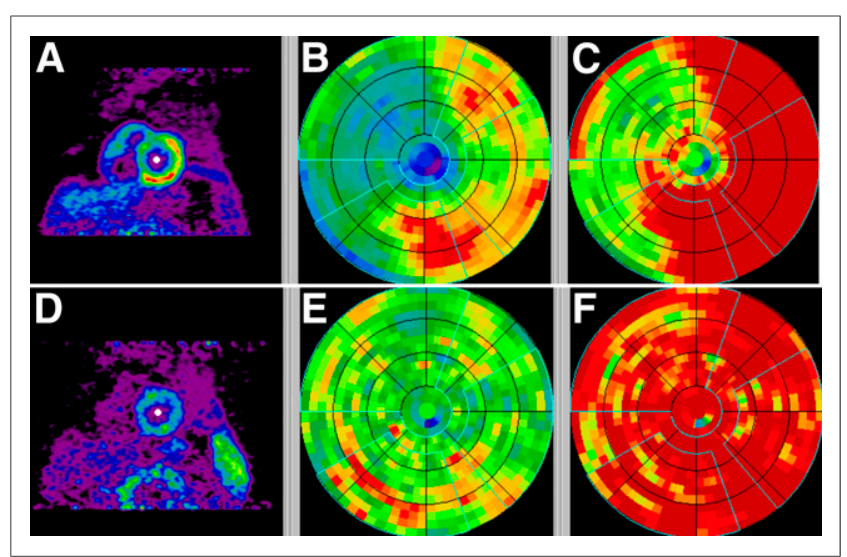

FIGURE 1. Polar maps in CAD patient with stress images $(A-C)$ and rest images (D-F) demonstrating reversible defect affecting mostly $L A D$ territory. Early summed images (0.5-2 min) were reoriented into shortaxis views ( $A$ and $D$ ), and polar maps were generated ( $B$ and $E$ ) and normalized on the basis of averages of healthy subjects ( $C$ and $F$ ). Vascular territories and left ventricular chamber were defined on polar map automatically.

with ensuing MFR is depicted as mean \pm SD (Fig. 3). There were no significant differences in flow between the LAD, LCx, and RCA territories in each condition, with $P$ values of 0.70 for resting MBF (RMBF), 0.11 for stress MBF (SMBF), and 0.99 for MFR. For each vascular territory, there was a significant difference between RMBF and SMBF, RMBF and MFR, and SMBF and MFR, with $P$ values less than 0.001 .

The regional MBF in the $\mathrm{LAD}, \mathrm{LCx}$, and RCA territories as well as global MBF derived from weighted contributions from each vascular territory (Eq. 5) in healthy subjects with low likelihood of myocardial ischemia, compared with regional MBF in $<50 \%$ stenosis and $\geq 50 \%$ stenosis vascular territories, is detailed in Table 1. In low-likelihood territories, mean \pm SD of global MBF was $0.73 \pm 0.13 \mathrm{~mL} / \mathrm{min} / \mathrm{g}$ at rest and $2.53 \pm 0.48$ $\mathrm{mL} / \mathrm{min} / \mathrm{g}$ at stress, leading to an MFR of $3.70 \pm 0.39$. In $<50 \%$ stenosis vascular territories, mean \pm SD of MBF was $0.73 \pm 0.09$ at rest and $2.02 \pm 0.40$ at stress, leading to an MFR of $2.97 \pm$ 0.76 . In $\geq 50 \%$ stenosis vascular territories, mean $\pm \mathrm{SD}$ of $\mathrm{MBF}$ was $0.86 \pm 0.21 \mathrm{~mL} / \mathrm{min} / \mathrm{g}$ at rest and $1.43 \pm 0.31 \mathrm{~mL} / \mathrm{min} / \mathrm{g}$ at stress, leading to an MFR of $1.86 \pm 0.59$. There was no significant difference in rest MBF in global or regional vascular flow between the low-likelihood and $<50 \%$ stenosis $(P=0.97)$ or $\geq 50 \%$ stenosis $(P=0.73)$ vascular territories. Differences in stress $\operatorname{MBF}(P=$ $0.004)$ and MFR $(P=0.003)$ between global flow in low-likelihood and $<50 \%$ vascular territories were significant. Similarly, differences in stress MBF $(P=0.001)$ and MFR $(P=0.001)$ between global flow in low-likelihood and $\geq 50 \%$ vascular territories were significant. Differences were also significant when comparing individual vascular territories from healthy subjects with individual vascular territories with $<50 \%$ stenosis or $\geq 50 \%$ stenosis from CAD patients (data not shown). Finally, when $<50 \%$ stenosis were compared with $\geq 50 \%$ stenosis vascular territories, there was no significant difference in rest $\mathrm{MBF}(P=0.073)$ but there was a significant difference in stress $\operatorname{MBF}(P<0.001)$ and MFR $(P<0.001)$.

The regional MBF and MFR in the vascular territories of healthy subjects with low likelihood of myocardial ischemia $(n=$ 21 territories) versus the vascular territories of CAD patients with $<50 \%$ stenosis ( $n=12$ territories) and $\geq 50 \%$ stenosis $(n=12$ territories) is illustrated further and presented as mean $\pm \mathrm{SD}$ 


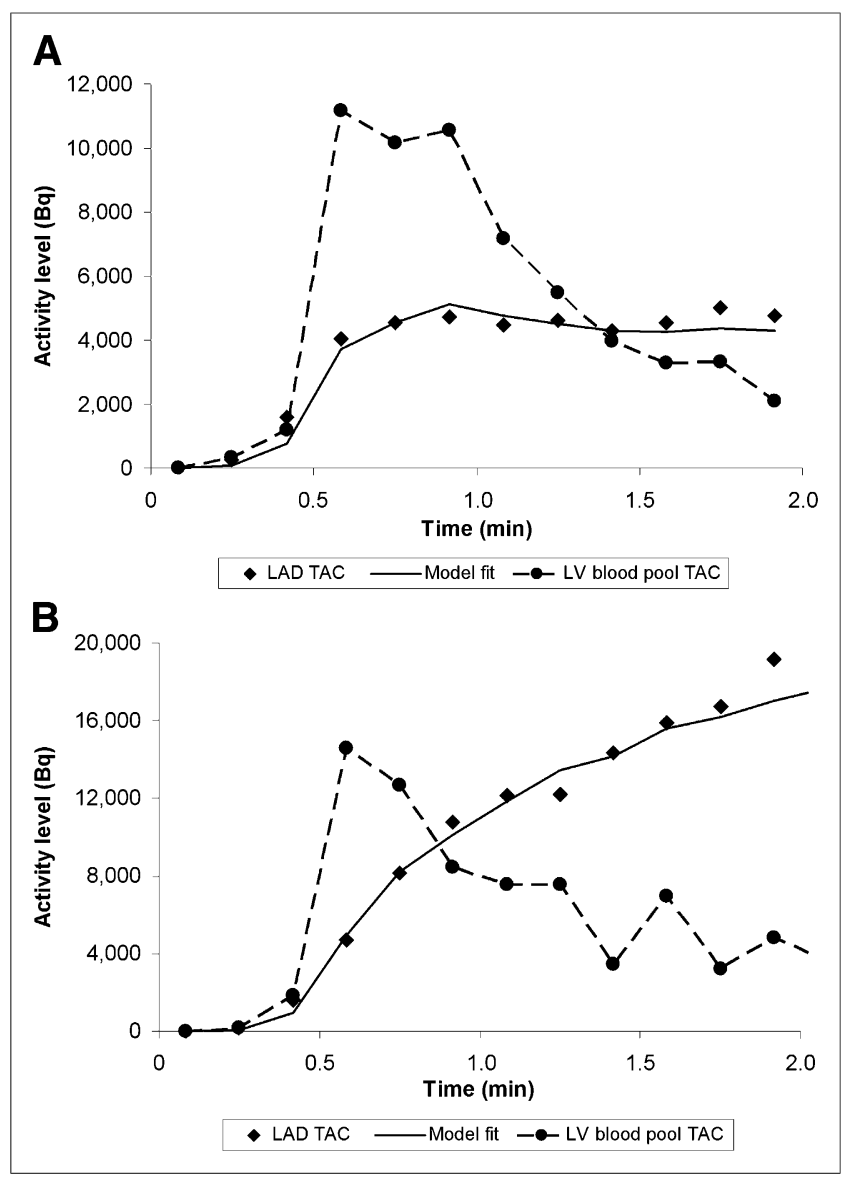

FIGURE 2. Left ventricular blood-pool time-activity curve $(\bullet)$ and LAD time-activity curve $(\bullet)$ obtained from predefined vascular ROI on polar map during rest $(A)$ and with adenosine stress $(B)$ in healthy subject. Solid curve is model fit to vascular territory myocardial time-activity curve based on single-compartment model.

(Fig. 4). There was no significant difference in RMBF between conditions. As detailed above, SMBF and MFR were significant when comparing low likelihood with $<50 \%$ stenosis, low likelihood with $\geq 50 \%$ stenosis, and $<50 \%$ stenosis with $\geq 50 \%$ stenosis vascular territories.

The scatterplot of the MFR obtained from each individual territory in healthy subjects with low likelihood of myocardial ischemia, compared with $<50 \%$ stenosis and $\geq 50 \%$ stenosis territories in CAD patients, is illustrated in detail in Figure 5 . Horizontal bars represent the respective mean values: 3.70 in low likelihood, 2.97 in $<50 \%$ stenosis, and 1.86 in $\geq 50 \%$ stenosis. Differences were significant when comparing the MFR from low likelihood with $<50 \%$ stenosis $(P=0.003)$, low likelihood with $\geq 50 \%$ stenosis $(P=0.001)$, and $<50 \%$ stenosis with $\geq 50 \%$ stenosis vascular territories $(P<0.001)$.

\section{DISCUSSION}

The present study is the first-in-human analysis of the absolute quantitation of $\mathrm{MBF}$ using flurpiridaz PET. Analyses were performed in both healthy subjects and CAD patients, providing MBF data over a wide range of conditions. The importance of the absolute quantitation of MBF and its prognostic implication in the presence of abnormal MFR has been previously established.
${ }^{15} \mathrm{O}$-water PET accurately detects CAD (12), and ${ }^{13} \mathrm{~N}$-ammonia MFR has a strong association with prognosis $(13,14) .{ }^{82} \mathrm{Rb} \mathrm{MBF}$ and MFR correctly detect 3-vessel CAD (15) and predict adverse cardiovascular events (16) beyond relative MPI and the sum stress score (17). Moreover, absolute quantitation of MBF with ${ }^{82} \mathrm{Rb}$ is an independent predictor of cardiac mortality in patients with known or suspected CAD and provides incremental risk stratification over established clinical variables and relative MPI (18).

Flurpiridaz exhibits many advantages over existing PET radiotracers and meets the conditions of an ideal perfusion tracer (2). Flurpiridaz has a high extraction fraction by the myocardium, a short positron range providing high-resolution images, and a relatively long half-life of $110 \mathrm{~min}$ allowing it to be produced at regional cyclotrons. This novel PET radiotracer is compatible with both pharmacologic and exercise stress imaging.

The present study demonstrates the feasibility of absolute quantitation of MBF with flurpiridaz. All MBF quantitation processes involve some assumptions, with potential errors introduced cancelling out when calculating MFR. Previous reference values using established PET radiotracers reported a rest MBF of $0.62-0.92 \mathrm{~mL} / \mathrm{min} / \mathrm{g}$ and a stress MBF of $1.97-3.55 \mathrm{~mL} / \mathrm{min} / \mathrm{g}$ in healthy subjects, usually leading to an MFR of 3.5-4.0. Patients with CAD display a significantly lower stress MBF typically leading to an MFR of 2.0-2.5 in borderline abnormal studies and $<2.0$ in frankly abnormal studies (1). MFR cutoffs for abnormal studies vary somewhat depending on the radiotracer used and the reference gold standard (fractional flow reserve during cardiac catheterization or $\geq 50 \%$ vs. $\geq 70 \%$ diameter stenosis during angiography) (19).

Because no reference standard was used in the present study, the accuracy of the quantitation values reported is subject to caution. The population was too small to use invasive angiography as a reference standard. Given the $94 \%$ myocardial extraction of flurpiridaz, we anticipate our absolute MBF quantitation results will correlate best with MBF values obtained with ${ }^{13} \mathrm{~N}$-ammonia and ${ }^{15} \mathrm{O}$-water, which exhibit high extraction fractions of $80 \%$ and $100 \%$, respectively, and have robust MBF reproducibility (1). We expect less correlation, however, with ${ }^{82} \mathrm{Rb}$-chloride because of its lower (65\%) and sigmoid-shaped extraction at elevated MBFs necessitating software correction (20). Future studies will be conducted to provide values of absolute MBF and ensuing MFR with cutoffs below which the presence of $\mathrm{CAD}$, whether epicardial or microvascular, may be predicted in a clinically useful manner.

Importantly, regional perfusion and myocardial uptake measurements are feasible with flurpiridaz PET imaging. In healthy subjects, a uniformity of MBF was observed at rest and during adenosine stress in the LAD, LCx, and RCA territories. Similar results have been observed with other radiotracers $(21,22)$.

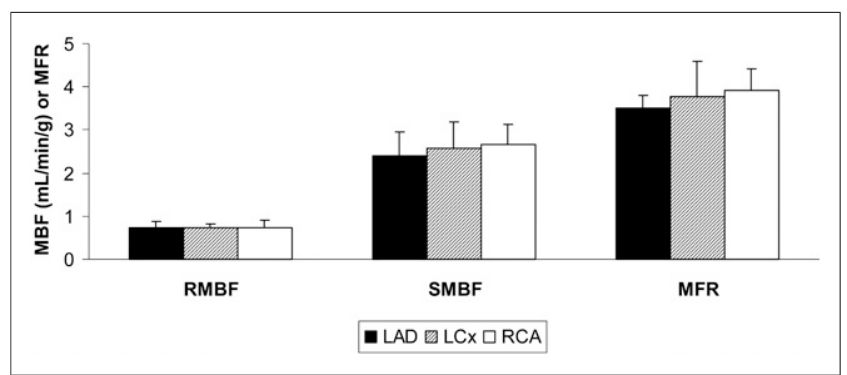

FIGURE 3. Regional MBF at rest (RMBF) and during adenosine stress (SMBF) and MFR in 3 vascular territories of healthy subjects. 
TABLE 1

MBF and MFR in Vascular Territories Characterized by Low Likelihood of Myocardial Ischemia, $<50 \%$ Stenosis, and $\geq 50 \%$ Stenosis

\begin{tabular}{|c|c|c|c|c|c|c|}
\hline \multirow[b]{2}{*}{ Flow } & \multicolumn{4}{|c|}{ Low likelihood ( $n=21$ territories from 7 subjects) } & \multirow{2}{*}{$\begin{array}{l}<50 \% \text { stenosis ( } n=12 \\
\text { territories from } 8 \text { patients) }\end{array}$} & \multirow{2}{*}{$\begin{array}{l}\geq 50 \% \text { stenosis ( } n=12 \\
\text { territories from } 8 \text { patients) }\end{array}$} \\
\hline & LAD & LCx & $\mathrm{RCA}$ & Global & & \\
\hline RMBF & $0.73 \pm 0.15$ & $0.73 \pm 0.09$ & $0.74 \pm 0.17$ & $0.73 \pm 0.13$ & $0.73 \pm 0.09$ & $0.86 \pm 0.21$ \\
\hline SMBF & $2.41 \pm 0.54$ & $2.58 \pm 0.60$ & $2.67 \pm 0.46$ & $2.53 \pm 0.48$ & $2.02 \pm 0.40$ & $1.43 \pm 0.31$ \\
\hline MFR & $3.50 \pm 0.29$ & $3.77 \pm 0.82$ & $3.93 \pm 0.47$ & $3.70 \pm 0.39$ & $2.97 \pm 0.76$ & $1.86 \pm 0.59$ \\
\hline \multicolumn{7}{|c|}{ Values are mean \pm SD. } \\
\hline
\end{tabular}

Interestingly, CAD vascular segments with $<50 \%$ stenosis exhibited stress MBF and MFR values between those from vascular segments with $\geq 50 \%$ stenosis and those from healthy subjects. Consistent with previous reports, this finding suggests that despite being labeled as angiographically nonsignificant, these territories are not normal and exhibit hemodynamic and metabolic abnormalities. Previously suggested mechanisms include abnormalities of endothelium-dependent or smooth muscle cell-dependent vasomotion, with these patients being at increased risk of future cardiovascular events (1).

The MBF quantitation procedure we adopted uses only the flurpiridaz kinetics of the first $1.5 \mathrm{~min}$. The procedure does not require taking blood samples from the patients to provide the input function and thus does not need cross-calibration of the PET measurement with other devices such as well counters that frequently introduce large errors in practical situations. The procedure is based on a few assumptions that are supported by known kinetics of flurpiridaz in blood and myocardium. First, it assumes that within the first $1.5 \mathrm{~min}$ after tracer injection there are few if any ${ }^{18} \mathrm{~F}$-labeled metabolites in the blood, which is supported by results in animal and human studies. Another assumption is that, after myocardial uptake of flurpiridaz, the compound is not cleared from the myocardium in a significant amount within $1.5 \mathrm{~min}$. This assumption is corroborated by animal and human studies demonstrating that the clearance rate constant of the compound in the myocardium was measured in hours $(3-6,9)$, and no additional clearance component (e.g., due to the interstitial space) of faster clearance rate was observed in the kinetics of the firstpass extraction fraction measurements (5). Thus, the modeling of the myocardial uptake of flurpiridaz as an irreversible process (Eq. 1 ) is quite valid. The 2 assumptions made here were also made by Sherif et al. (7) in their calculations of MFR and the tracer retentions in the myocardium of pig models, except that they calculated the retentions using a longer scan time (5-10 min for myocardial activity and 0-3 min for area under the blood time-activity curve). The longer times made the assumptions less valid. Although the errors created were somewhat cancelled when calculating the MFR (as a ratio of stress and rest retentions), the retention measurements they obtained did not correlate well with the MBF determined by microspheres.

Another reason for their retention measurements failing to match the microsphere-measured perfusion is that their retention measurements did not consider partial-volume and spillover effects in their calculations. In the procedure used in the present study, the partial-volume effect and spillover of activities were explicitly accounted for (Eq. 2), although some assumptions and approximations (Eqs. 3 and 4) were used to simplify the procedure and make the estimation of myocardial perfusion more tractable and robust. The approach proposed by Nekolla et al. (6) using a 2tissue-compartment did account for the partial-volume effect and spillover fraction in a manner similar to our procedure. However, their approach using 10 or $20 \mathrm{~min}$ of kinetics (0-10 or 0-20 min) is susceptible to subject movement during the imaging time that is difficult to avoid, especially for stress studies. Also, the assumption of having negligibly labeled metabolites in the blood is no longer valid for flurpiridaz over such a long period.

A potential disadvantage of using short kinetics to quantitate perfusion is the lack of adequate count statistics to give a reliable assessment, such as with ${ }^{82} \mathrm{Rb}$-determined MBF. However, because of the high first-pass extraction fraction of flurpiridaz by the myocardium, the dynamic PET images of the heart in the first 2 min were of adequate quality and allowed good measurement kinetics in myocardial regions (Fig. 2). The use of modeling fitting to the measured kinetics rather than just use of a single integrated value also maximizes the value of the available data for estimation of myocardial perfusion. In the current study, the relative small variability of the MBF estimates obtained under both rest and stress conditions in healthy subjects and in the myocardial segments of $<50 \%$ stenosed CAD patients clearly demonstrate the stability of the measurements based on the 1.5-min short kinetics and support the robustness of the quantitation procedure used.

In our MBF quantitation procedure, we also used a fixed value of 0.94 for the first-pass extraction fraction. This first-pass extraction fraction value was previously determined in a Langendorff preparation of rat heart (5) and was found to be independent of perfusion flow. Although the first-pass extraction fraction was taken directly from an animal study, its use in the present study

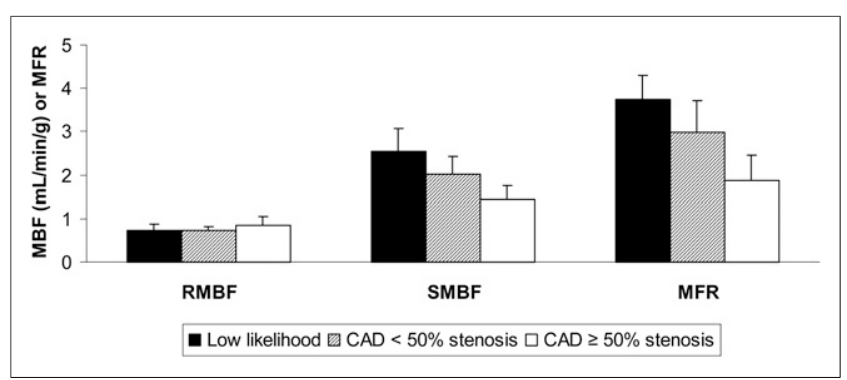

FIGURE 4. Regional MBF at rest (RMBF) and during adenosine stress (SMBF) and MFR in vascular territories of healthy subjects with low likelihood of myocardial ischemia vs. vascular territories of CAD patients with $<50 \%$ stenosis and $\geq 50 \%$ stenosis. 


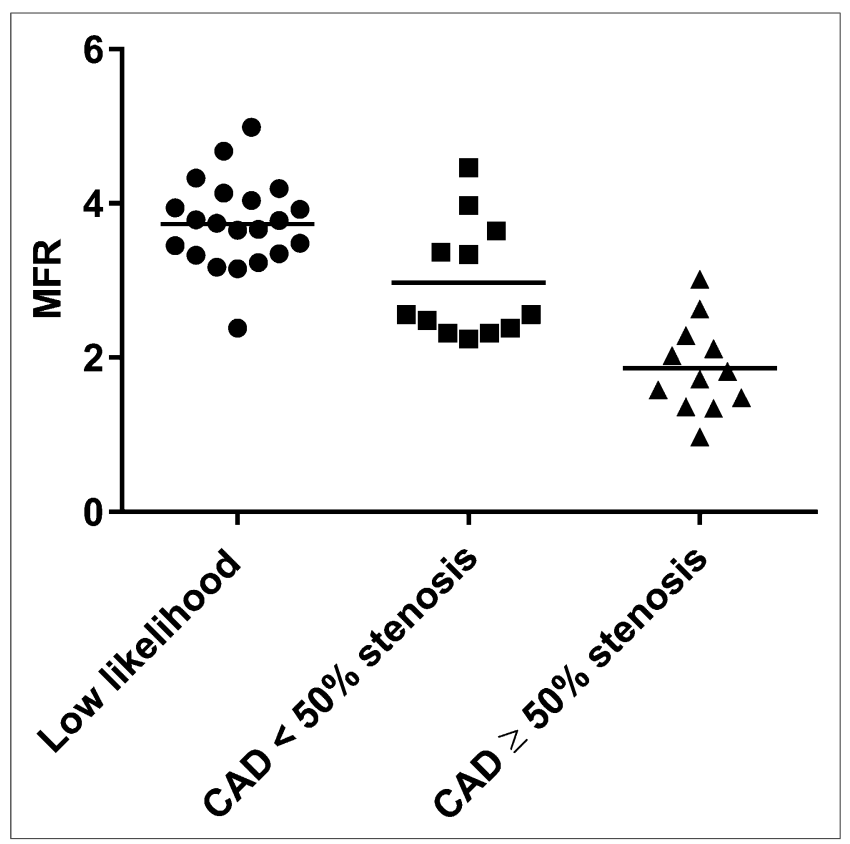

FIGURE 5. Scatterplot of MFR obtained from each individual territory in healthy subjects with low likelihood of myocardial ischemia vs. CAD patients with $<50 \%$ stenosis and $\geq 50 \%$ stenosis.

gives human MBF values comparable to the commonly accepted values obtained by other methods. Even though the extraction fraction in humans may deviate slightly from 0.94 , the resulting MBF would not change significantly. In addition, a high first-pass extraction fraction is not significantly dependent on perfusion, and hence the conversion of tracer uptake to MBF can be greatly simplified (Eq. 6).

Certain aspects of the current MBF quantitation procedure, however, can be further improved to make it more useful in the clinical setting. For example, the extension of the method for generating MBF parametric images could take the quantitation to a level usable for routine clinical studies. Subsequent studies will measure MBF over finer coronary segments such as the standardized 17-segment left ventricular myocardial model, which may be of greater clinical use. In the present study, healthy subjects were recruited on the basis of their low likelihood of having myocardial ischemia. Given the presence of classic cardiovascular risk factors (Table 2) in this group, and despite their absence of angina, anginal equivalent, or evidence of stress-inducible ischemia by electrocardiogram or PET MPI, this group may have had subclinical CAD. This may theoretically have led the stress MBF in the group of healthy subjects to be lower than in a group of young subjects with complete absence of cardiovascular risk factors, which were not included in the present study. Also, comparison of MBF measurements obtained through flurpiridaz with those currently accepted using ${ }^{13} \mathrm{~N}$-ammonia PET in the same subjects is expected to provide further validation or improvement of the quantitative MBF method reported in this study, for example, confirmation of the first-pass extraction fraction of 0.94 .

\section{CONCLUSION}

With a robust perfusion quantitation procedure, absolute quantitation of MBF using Flurpiridaz F 18 cardiac PET imaging is feasible in both healthy subjects with low likelihood of
TABLE 2

Baseline Characteristics of Healthy Subjects with Low Likelihood of Myocardial Ischemia and CAD Patients

\begin{tabular}{|c|c|c|}
\hline Characteristic & $\begin{array}{l}\text { Healthy } \\
\text { subjects } \\
(n=7)\end{array}$ & $\begin{array}{c}\text { CAD } \\
\text { patients } \\
(n=8)\end{array}$ \\
\hline Age (y) & 66 & 68 \\
\hline \multicolumn{3}{|l|}{ Sex $(\%)$} \\
\hline Male & 86 & 100 \\
\hline Female & 14 & 0 \\
\hline Body mass index $\left(\mathrm{kg} / \mathrm{m}^{2}\right)$ & 28 & 27 \\
\hline \multicolumn{3}{|c|}{ Cardiovascular risk factors (\%) } \\
\hline Hypertension & 43 & 75 \\
\hline Diabetes mellitus & 0 & 50 \\
\hline Dyslipidemia & 71 & 88 \\
\hline \multicolumn{3}{|l|}{ Tobacco use } \\
\hline Current & 0 & 0 \\
\hline Former & 43 & 75 \\
\hline Never & 57 & 25 \\
\hline \multicolumn{3}{|l|}{ Labs (mg/dL) } \\
\hline Low-density lipoprotein & 97 & 86 \\
\hline High-density lipoprotein & 52 & 44 \\
\hline Creatinine & 1.0 & 1.0 \\
\hline \multicolumn{3}{|l|}{ Medications (\%) } \\
\hline Antiplatelet agent & 86 & 88 \\
\hline Statin & 71 & 88 \\
\hline $\begin{array}{l}\text { Angiotensin-converting } \\
\text { enzyme inhibitor/ } \\
\text { angiotensin } \\
\text { receptor blocker }\end{array}$ & 43 & 63 \\
\hline Beta-blocker & 57 & 75 \\
\hline
\end{tabular}

Values are expressed as means or percentages.

myocardial ischemia and CAD patients. No significant differences in MBF (either at rest or with adenosine stress) and MFR were found among the 3 coronary perfusion territories in subjects with no stress-inducible ischemia. In CAD patients, diseased vascular segments had significantly lower MBF in response to adenosine stress and thus a reduced MFR. Future studies will determine cutoff values for stress MBF and MFR in patients with CAD for direct clinical applicability. We believe patient risk stratification using PET imaging will be feasible in a broad clinical setting using both relative perfusion and the absolute quantitation of MBF. These initial results suggest that flurpiridaz, which is not yet approved by the U.S. Food and Drug Administration as a PET MPI agent, is uniquely poised as a radiotracer to fulfill all these requirements.

\section{DISCLOSURE}

The costs of publication of this article were defrayed in part by the payment of page charges. Therefore, and solely to indicate this fact, this article is hereby marked "advertisement" in accordance with 18 USC section 1734 . The phase II clinical trial analyzing 
flurpiridaz MPI was sponsored by Lantheus Medical Imaging. Lantheus Medical Imaging had no role in the design of this study, analysis of its data, or writing of the manuscript. The study is supported by NIH grant T32 HL007895 and a research grant from Lantheus Medical Imaging. Dr. Maddahi is a scientific advisor to Lantheus Medical Imaging. No other potential conflict of interest relevant to this article was reported.

\section{ACKNOWLEDGMENTS}

We are indebted to James Sayre, $\mathrm{PhD}$, from the UCLA Department of Biostatistics who performed the statistical analyses. We are also grateful to John Williams and David Truong for their expert technical assistance.

\section{REFERENCES}

1. Schindler TH, Schelbert HR, Quercioli A, Dilsizian V. Cardiac PET imaging for the detection and monitoring of coronary artery disease and microvascular health. JACC Cardiovasc Imaging. 2010;3:623-640.

2. Maddahi J. Properties of an ideal PET perfusion tracer: new PET tracer cases and data. J Nucl Cardiol. 2012;19(suppl 1):S30-S37.

3. Yalamanchili P, Wexler E, Hayes M, et al. Mechanism of uptake and retention of F-18 BMS-747158-02 in cardiomyocytes: a novel PET myocardial imaging agent. J Nucl Cardiol. 2007;14:782-788.

4. Yu M, Guaraldi MT, Mistry M, et al. BMS-747158-02: a novel PET myocardial perfusion imaging agent. J Nucl Cardiol. 2007;14:789-798.

5. Huisman MC, Higuchi T, Reder S, et al. Initial characterization of an ${ }^{18} \mathrm{~F}$-labeled myocardial perfusion tracer. J Nucl Med. 2008;49:630-636.

6. Nekolla SG, Reder S, Saraste A, et al. Evaluation of the novel myocardial perfusion positron-emission tomography tracer ${ }^{18} \mathrm{~F}-\mathrm{BMS}-747158-02$ : comparison to ${ }^{13} \mathrm{~N}$-ammonia and validation with microspheres in a pig model. Circulation. 2009;119:2333-2342.

7. Sherif HM, Nekolla SG, Saraste A, et al. Simplified quantification of MFR with flurpiridaz F 18: validation with microspheres in a pig model. $\mathrm{J} \mathrm{Nucl} \mathrm{Med}$. 2011;52:617-624.

8. Sherif HM, Saraste A, Weidl E, et al. Evaluation of a novel ${ }^{18} \mathrm{~F}$-labeled positronemission tomography perfusion tracer for the assessment of myocardial infarct size in rats. Circ Cardiovasc Imaging. 2009;2:77-84.
9. Maddahi J, Czernin J, Lazewatsky J, et al. Phase I, first-in-human study of BMS747158, a novel ${ }^{18}$ F-labeled tracer for myocardial perfusion PET: dosimetry, biodistribution, safety, and imaging characteristics after a single injection at rest. J Nucl Med. 2011;52:1490-1498.

10. Berman DS, Maddahi J, Tamarappoo BK, et al. Phase II safety and clinical comparison with single-photon emission computed tomography myocardial perfusion imaging for detection of coronary artery disease: flurpiridaz $\mathrm{F} 18$ positron emission tomography. J Am Coll Cardiol. 2013;61:469-477.

11. Schindler TH, Zhang XL, Vincenti G, et al. Diagnostic value of PET-measured heterogeneity in myocardial blood flows during cold pressor testing for the identification of coronary vasomotor dysfunction. J Nucl Cardiol. 2007;14:688-697.

12. Kajander S, Joutsiniemi E, Saraste M, et al. Cardiac positron emission tomography/computed tomography imaging accurately detects anatomically and functionally significant coronary artery disease. Circulation. 2010;122:603-613.

13. Tio RA, Dabeshlim A, Siebelink HM, et al. Comparison between the prognostic value of left ventricular function and myocardial perfusion reserve in patients with ischemic heart disease. J Nucl Med. 2009;50:214-219.

14. Herzog BA, Husmann L, Valenta I, et al. Long-term prognostic value of ${ }^{13} \mathrm{~N}$ ammonia myocardial perfusion positron emission tomography added value of coronary flow reserve. J Am Coll Cardiol. 2009;54:150-156.

15. Parkash R, deKemp RA, Ruddy TD, et al. Potential utility of rubidium 82 PET quantification in patients with 3-vessel coronary artery disease. J Nucl Cardiol. 2004;11:440-449.

16. Fukushima K, Javadi MS, Higuchi T, et al. Prediction of short-term cardiovascular events using quantification of global myocardial flow reserve in patients referred for clinical ${ }^{82} \mathrm{Rb}$ PET perfusion imaging. J Nucl Med. 2011;52:726-732.

17. Ziadi MC, Dekemp RA, Williams KA, et al. Impaired myocardial flow reserve on rubidium-82 positron emission tomography imaging predicts adverse outcomes in patients assessed for myocardial ischemia. J Am Coll Cardiol. 2011;58:740-748.

18. Murthy VL, Naya M, Foster CR, et al. Improved cardiac risk assessment with noninvasive measures of coronary flow reserve. Circulation. 2011;124:2215-2224.

19. Johnson NP, Gould KL. Integrating noninvasive absolute flow, coronary flow reserve, and ischemic thresholds into a comprehensive map of physiological severity. JACC Cardiovasc Imaging. 2012;5:430-440.

20. Saraste A, Kajander S, Han C, Nesterov SV, Knuuti J. PET: is myocardial flow quantification a clinical reality? J Nucl Cardiol. 2012;19:1044-1059.

21. Czernin J, Muller P, Chan S, et al. Influence of age and hemodynamics on myocardial blood flow and flow reserve. Circulation. 1993;88:62-69.

22. Nagamachi S, Czernin J, Kim AS. Reproducibility of measurements of regional resting and hyperemic myocardial blood flow assessed with PET. J Nucl Med. 1996;37:1626-1631. 